\title{
MINUTE FOREIGN MATERIAL ANALYSIS USING MONO-CAPILLARY WITH 10-MICROMETER SPATIAL RESOLUTION
}

\author{
Sumito Ohzawa, ${ }^{*}$ Yoshihiro Yokota,* Daisuke Matsunaga,* Shintaro Komatani,* Kenichi \\ Obori,* Andrew Whitley** and Sergey Mamedov** \\ * HORIBA Ltd, 2 Miyanohigashi, Kissyoin, Minami-ku, Kyoto 601-8510, Japan \\ ** HORIBA Jobin Yvon Inc., 3880 Park Avenue Edison, New Jersey USA 08820
}

The scanning X-ray microscope is an analysis device that irradiates a fine X-ray beam on the sample, measures fluorescent X-rays and transmitted X-rays that occur from the sample while scanning, and obtains fluorescent and transmitted X-ray images. It includes the following features - atmospheric analysis (no vacuum required), no requirement for sample pre-treatment and non-destructive analysis (all in contrast with SEM-EDX that must be performed in a vacuum, and often requires a conductive coating). In addition, because of the transmission of X-rays through the sample, analysis can be made inside without exposing the area of interest. The spatial resolution of the scanning Xray microscope depends on the diameter of the X-ray beam. We use a scanning X-ray microscope with mono-capillary X-ray guide tube which has the narrowest X-Ray beam available. The diameter of this X-ray beam is 10 micrometers.

Recent analysis requirements have become smaller and smaller and more complicated due to the development of functional and composite materials and high-density electronic and electrical parts. Foreign objects mixed into materials, which cause defective products, are sometimes located not only at the surface but also internally in materials, and it can be a non-trivial job to manually locate small foreign objects and extract them. The X-ray microscope with mono-capillary provides high resolution transmitted X-ray images with little or no distortion from the surface to the inside of the sample. Therefore it is easy to find and analyze foreign objects without extracting them. Importantly, optical, X-ray fluorescence and transmitted X-ray images are available all together, making analysis and interpretation easy. Due to these features, the X-ray microscope is suitable for foreign object analysis of electrictronic parts, medicine, food products and articles for daily use, with the added advantage that no sample preparation is necessary. This instrument is already proving useful for the quality improvement of many products. 


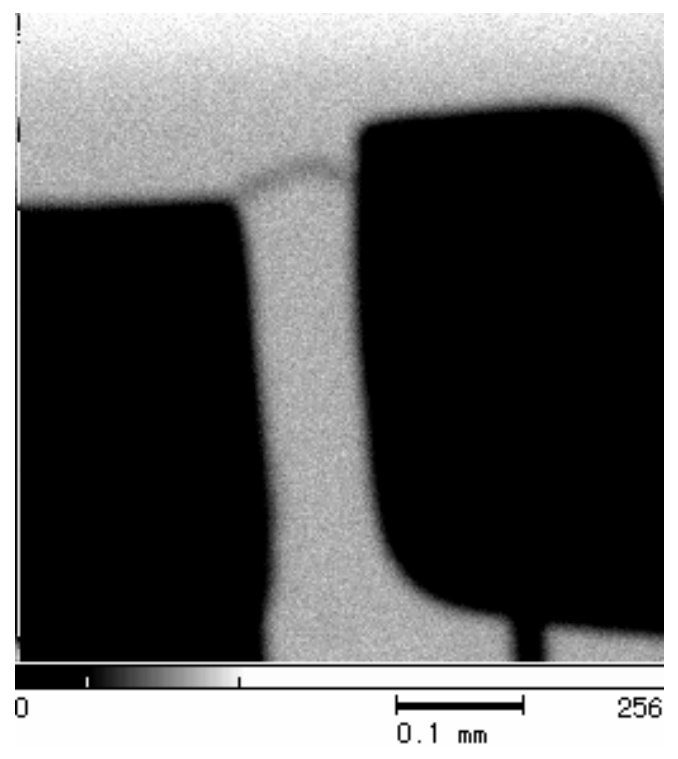

Figure 1. Transmission image of electronic device.

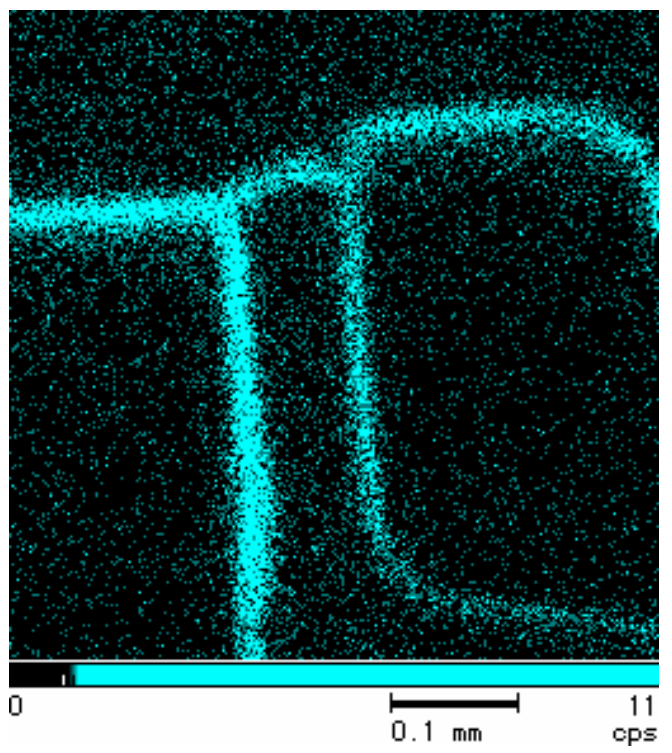

Figure 2. $\mathrm{Ag} \mathrm{K}_{\alpha}$ chemical image of electronic device.

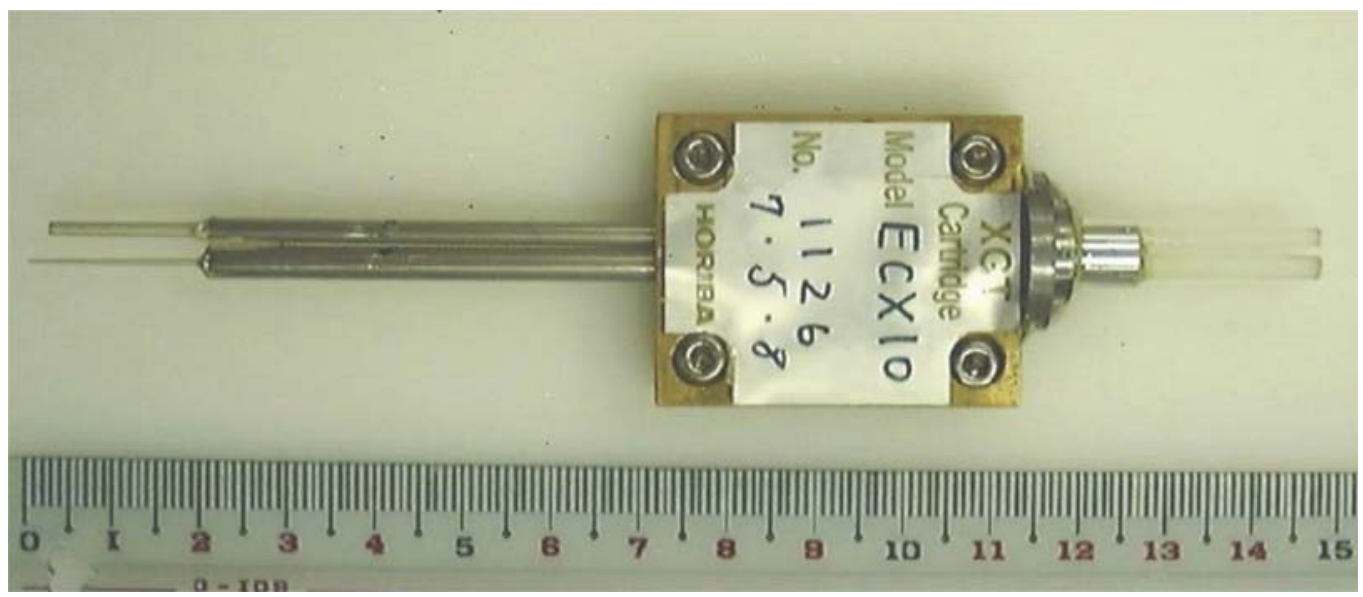

Figure 3. XGT-5000 cartridge with two mono capillaries. 